\title{
Interferon- $\alpha$ prevents apoptosis of endothelial cells after short-term exposure but induces replicative senescence after continuous stimulation
}

\author{
Johannes Pammer ${ }^{1}$, Christine Reinisch ${ }^{2}$, Peter Birner ${ }^{1}$, Kristin Pogoda ${ }^{3}$, Michael Sturzl ${ }^{3,4}$ \\ and Erwin Tschachler ${ }^{2,5}$ \\ ${ }^{1}$ Institute of Clinical Pathology, Medical University of Vienna, Vienna, Austria; ${ }^{2}$ Department of Dermatology, \\ Medical University of Vienna, Vienna, Austria; ${ }^{3}$ GSF-National Research Center for Environment and Health, \\ Institute of Molecular Virology, Neuherberg, Germany; ${ }^{4}$ Division of Molecular and Experimental Surgery, \\ Department of Surgery, University of Erlangen, Erlangen, Germany and ${ }^{5}$ Centre de Recherches et \\ d́Investigation Epidermiques et Sensorielles (CE.R.I.E.S.), Neuilly, France
}

\begin{abstract}
Although the antiangiogenic activity of type I interferons (IFN) is well known, the mechanism by which it occurs is unclear. In the present study, we have investigated effects of short-term and long-term IFN- $\alpha$ exposure on different types of endothelial cells (EC). Short-term IFN- $\alpha$ treatment resulted in a distinct reduction of apoptosis of serum and growth factor starved HUVEC and HDMEC. This was accompanied by a strong upregulation of the IFN inducible guanylate binding protein-1 (GBP-1) whereas no consistent regulation of several known antiapoptotic proteins was evident. Stable transfection of HUVEC with an expression vector for GBP-1 mimicked the protective effect of IFN- $\alpha$, suggesting that GBP-1 may contribute to the inhibition of apoptosis. When IFN- $\alpha$, together with serum and EC growth factors, was present continuously a decrease of population doublings by more than $40 \%$ was observed in both HDMEC and HCAEC. In addition, the cells displayed a senescent phenotype significantly earlier than control cells and showed an increased adherence for monocytes. Our findings suggest that the antiangiogenic effect of IFN- $\alpha$ is mediated by inducing EC senescence rather than EC apoptosis. Furthermore IFN- $\alpha$ released in chronic inflammatory conditions might contribute via its prosenescent activity to the pathogenesis of atherosclerosis.
\end{abstract}

Laboratory Investigation (2006) 86, 997-1007. doi:10.1038/labinvest.3700461; published online 7 August 2006

Keywords: apoptosis; endothelium; GBP-1; interferon-alpha; senescence

Type I interferons (IFNs) are immunomodulatory cytokines that are upregulated during viral infections. ${ }^{1}$ In contrast to IFN- $\beta$ which is a single protein produced by lymphocytes, ${ }^{1}$ IFN- $\alpha$ comprises about 20 structurally related proteins which are primarily produced by monocytes ${ }^{1}$ and plasmacytoid dendritic cell (PDC) precursors. ${ }^{2}$ However, during viral and chlamydial infection or after stimulation with immunomodulators, ${ }^{3}$ type I IFNs are also produced by a variety of other cell types including $\mathrm{EC},{ }^{4}$ smooth muscle cells ${ }^{5}$ and keratinocytes. ${ }^{6}$ IFN- $\alpha$ inhibits viral replication and supports virus clearance in infected cells in an autocrine and paracrine

Correspondence: Dr E Tschachler, MD, Department of Dermatology, Medical University of Vienna, Waehringer Guertel 18-20, 1090 Vienna, Austria.

E-mail: erwin.tschachler@meduniwien.ac.at

Received 22 July 2005; revised and accepted 30 June 2006; published online 7 August 2006 fashion. Therapeutically, IFN- $\alpha$ is used in the treatment of Hepatitis $\mathrm{C}^{7}$ and in antitumor therapy, ${ }^{8,9}$ and for its antiangiogenic activity in the treatment of recalcitrant hemangiomas. ${ }^{10}$

Besides in clinical practice, the antiangiogenic effect of IFN- $\alpha$ has been well established in animal models: high-dose and long-term treatment inhibits neoangiogenesis of solid tumors ${ }^{11,12}$ and retards wound healing. ${ }^{13}$ This antiangiogenic action of type I IFNs is thought to be primarily due to their antiproliferative activity on EC. ${ }^{14-17}$ However, the antiproliferative activity of IFNs is not generally accepted and is challenged by data of several investigators. ${ }^{18-20}$ Some authors have even reported an increased proliferation of microvascular EC after IFN- $\alpha_{2 \mathrm{a}}$ treatment. ${ }^{14,20,21}$ Another explanation for the antiangiogenic activity of IFNs was suggested by Sgonc et $a l,{ }^{22}$ who reported that IFN- $\alpha$ may induce apoptosis of EC. However, confirmation of these data by others is still pending. 
These contradictory data prompted us to study the effects of short-term and long-term IFN- $\alpha$ treatment on different types of EC in vitro. We demonstrate that short-term IFN- $\alpha$ treatment under conditions of serum starvation inhibits EC apoptosis whereas long-term treatment of cells in the presence of growth factors and serum induces a sencescent phenotype. As these senescent EC also showed an increased adherence of blood mononuclear cells our data suggest that continuous exposure to IFN- $\alpha$, as it occurs in chronic inflammatory disorders, might be one of the factors able to trigger and/or aggravate atherosclerosis.

\section{Methods}

\section{Cell Culture and Reagents}

Human umbilical vein (HUVEC) endothelial cells were isolated and characterized as described previously $^{23}$ and human dermal microvascular (HDMEC) and human coronary arterial (HCAEC) endothelial cells were purchased from CellSystems (St Katharinen, Germany). Stable transduction of HUVEC with GBP-1 was reported previously. ${ }^{24}$ HUVEC of the same passage transduced with an empty control vector served as a negative control. All cells were serially cultured on gelatine-coated plastic culture dishes in endothelial cell growth medium (EGM) or endothelial cell growth medium EBM-2 including endothelial cell growth serum (CellSystems). All experiments with EC were initiated between passages 4 and 8. Experiments were performed in either M199 medium, ${ }^{23}$ basal medium lacking both serum and growth factors (short-term exposure, antiapoptotic experiments) or in fully supplemented medium (long-term exposure, senescence experiments) with either INTRON ${ }^{\circledR}-A$ (Interferon $\alpha-2 b$, Schering, Kenilworth, NY, USA) or ROFERON ${ }^{\circledR}$-A (Interferon $\alpha$-2a, Hoffmann-La Roche Inc, Nutley, NJ, USA) at the concentrations indicated.

Population doublings were counted as described. ${ }^{25}$ Apoptosis of EC was assessed by typical morphological changes observed by inverted light microscopy as well as by using a cell death detection ELISA measuring histone released in the supernatant (Roche, Mannheim, Germany). Ki-67 (clone MIB-1, Dako, Glostrup, Denmark) and podoplanin ${ }^{26}$ expression were evaluated on EC grown on Lab-Tek Chamber Slides (Nalge Nunc, Naperville, IL, USA) by immunohistochemistry. $\beta$-Galactosidase staining of EC grown on Lab-Tek Chamber Slides was performed as described by Dimri et al. ${ }^{27}$

\section{Western Blotting}

Subconfluent endothelial cell monolayers were incubated in the presence or absence of IFN- $\alpha$ for 20-50 min for AKT phosphorylation experiments and $18-24 \mathrm{~h}$ for all other experiments. At the end of the incubation, cells were harvested and lysed in NP-40 buffer (1\% NP-40 in PBS) containing $1 \mathrm{mM}$ PMSF and $100 \mu \mathrm{M}$ sodium orthovanadate (Sigma). Protein concentrations were measured by a Micro BCA Protein Assay Reagent (Pierce, Rockford, IL, USA). Lysates were subjected to SDS-PAGE in $12 \%$ gels. Prior to SDS-PAGE, lysates were mixed with reducing SDS-loading buffer $(7.2 \%$ SDS, $9 \mathrm{mmol} / \mathrm{l}$ EDTA, $20 \%$ glycerol, $10 \mathrm{mmol} / \mathrm{l}$ dithiothreitol, $13 \mathrm{mmol} / \mathrm{l}$ Tris-phosphate buffer, $\mathrm{pH}$ 6.8) and boiled for $5 \mathrm{~min}$. Separated proteins were then electroblotted onto a nitrocellulose membrane (Schleicher \& Schüll, Germany) at $0.8 \mathrm{~mA} / \mathrm{cm}^{2}$ for $2 \mathrm{~h}$. The membranes containing the complete set of samples were blocked in blocking buffer (5\% nonfat dry milk in PBS) followed by immune overlay with the following antibodies: RIG-G (clone 25, BD Biosciences, Heidelberg, Germany), p21 (Ab-1, Oncogene, Cambridge, MA, USA), p27 (clone SX53G8, Dako, Glostrup, Denmark), p53 (Immunotech, Marseille, France), Cyclin D1 (Ventana, Tucson, AZ, USA), Bak (TC-100, Calbiochem, Cambridge, MA, USA), Mcl-1 (Dako), Bcl-2 (bcl-2-124, Dako), bax (Ab-1, Calbiochem) and bcl-x (Transduction Laboratories, Lexington, KY, USA), Id-1 (Santa Cruz Biotechnology, Santa Cruz, CA, USA), polyclonal rabbit $^{24}$ or monoclonal rat anti-GBP-1 (1B1 $\left.{ }^{28}\right)$, AKT (Cell Signalling, Beverly, MA, USA) and pAKT (Ser473, Cell Signalling). After washing, bound antibodies were detected with HRP-labelled sheep anti mouse-IgG (Amersham Life Science, UK) or HRP-labelled goat anti-rabbit IgG (Pierce, Rockford, IL, USA), respectively. After rinsing in Western blotting detection reagent ECL (Amersham), membranes were exposed to a X-OMAT-AR film (Eastman Kodak, NY, USA) for 5, 30 and $60 \mathrm{~min}$ each. Western blot bands of Id-1 and GBP-1 were quantitated by GelPro Analyzer 4.0 (Media cybernetics).

\section{Isolation of Monocytes from PBMNC}

Peripheral blood mononuclear cells (PBMNC) were prepared from peripheral venous blood of healthy volunteers after anticoagulation with $1.8 \mathrm{mg}$. EDTA/ ml blood. After Ficoll-Paque (Pharmacia, Uppsala, Sweden) density gradient centrifugation, PBMNC were collected and washed three times with HBSS (Gibco, Invitrogen Corporation, Carlsbad, CA, USA). Positive selection of monocytes was performed by adding MACS colloidal super-paramagnetic microbeads conjugated with anti-human CD14 antibodies (Miltenyi Biotec, Bergisch Gladbach, Germany) to cooled, freshly prepared PBMNC preparations in MACS buffer (PBS (Gibco, Invitrogen Corporation, Carlsbad, CA, USA) with $5 \mathrm{mM}$ EDTA and $0.5 \%$ bovine serum albumin (SigmaAldrich, St Louis, MO, USA)) according to the manufacturer's instructions. Cells and microbeads were incubated for $15 \mathrm{~min}$ at $4-6^{\circ} \mathrm{C}$. In the mean- 
time, the separation column (Miltenyi Biotec) was positioned in the magnetic cell separator MidiMACS and washed with MACS buffer at room temperature. The cells were washed with MACS buffer, resuspended, and loaded onto the top of the separation column. The eluent containing CD14 cells was discarded and after removal of the column from the magnet, trapped monocytes $\left(\mathrm{CD} 14^{+}\right)$were eluted with cold MACS buffer, centrifuged, counted and resuspended at a concentration of $1 \times 10^{7}$ cells in $1 \mathrm{ml}$ HBSS. Cells were loaded with $10 \mu \mathrm{M} / \mathrm{ml}$ carboxyfluorescein diacetate (CFDA, Molecular Probes, Eugene, OR, USA) for $15 \mathrm{~min}$ at $37^{\circ} \mathrm{C}$, washed, centrifuged and finally resuspended at a concentration of $2 \times 10^{6}$ cells $/ \mathrm{ml}$ endothelial cell medium (EBM-2).

\section{Cell Adhesion Experiments}

HCAEC (CellSystems) and HDMEC of different population doublings were seeded on coverslips placed in tissue culture plates $\left(\right.$ Falcon $^{\mathrm{TM}}$, Becton Dickinson Labware, Franklin Lakes, NJ, USA) and grown to confluence. Monolayers were washed twice with EBM-2 and incubated with 500000 CFDA loaded monocytes $/ \mathrm{cm}^{2}$ for $2 \mathrm{~h}$ at $37^{\circ} \mathrm{C}, 5 \%$ $\mathrm{CO}_{2}$. The plates were shaken every $15 \mathrm{~min}$. After the incubation period, monolayers were washed three times with EBM-2 to remove nonadherent monocytes and fixed with $4 \%$ paraformaldehyde. Coverslips were mounted on glass slides using fluorescent mounting medium (Fluoprep, bioMérieux, Marcyl'Etoile, France) and analyzed on a conventional fluorescent microscope (Olympus Provis, Vienna, Austria).

\section{Statistical Analysis}

The statistical significance of intergroup differences was evaluated by univariate analysis of variance (ANOVA) and subsequent Tukey-tests. As a control, Wilcoxon tests were performed as appropriate for additional comparison of groups. A two-tailed $P$ value of $\leq 0.05$ was considered statistically significant. SPSS 10.0.7 (SPSS Inc., Chicago, IL, USA) was used for analysis.

Data are presented as the mean value \pm s.e. for each group.

\section{Results}

Type I Interferon Enhances Survival of HUVEC and HDMEC after Growth Factor Withdrawal

To test the effects of IFN- $\alpha$ on EC survival and proliferation in vitro, we exposed HUVEC and HDMEC to $1000 \mathrm{IU} / \mathrm{ml} \mathrm{IFN}-\alpha$ in the presence and absence of serum and EC growth factors. As expected and reported previously ${ }^{29}$ growth factor and serum withdrawal had a dramatic effect on both HUVEC and HDMEC as compared to cells grown under standard conditions: as early as $48 \mathrm{~h}$ after serum withdrawal, and more pronounced at $96 \mathrm{~h}$, a large proportion of cells exhibited morphological signs of apoptosis and had detached from the bottom of the culture vessel (Figure 1c and d). This effect could be prevented when the culture media were supplemented with IFN- $\alpha$ (Figure $1 \mathrm{e}$ and f). The prevention of apoptosis by IFN- $\alpha$ was also demonstrated by a significant reduction of spontaneous histone release in IFN-treated cultures as compared to untreated ones (Figure 2a), and by a significantly slower reduction of cell numbers in treated as compared to untreated cells (Figure 2b). As demonstrated by a comparable proportion of Ki67 positive cells IFN- $\alpha$ did not induce proliferation of EC (Figure 2c). No significant effect of IFN- $\alpha$ on cell numbers and cell survival was observed when EC were grown in medium supplemented with EC growth factors (data not shown). These data demonstrate that IFN- $\alpha$ treatment prevents EC apoptosis after serum and growth factor withdrawal but does not induce EC proliferation in vitro at the concentrations used.

\section{Interferon- $\alpha$ Downregulates Id-1 and Upregulates GBP-1 Expression in EC}

To investigate whether expression of proteins involved in cell survival was modulated, we tested EC lysates after IFN- $\alpha$ exposure by Western blot analysis. As a positive control for IFN- $\alpha$-activity, the expression of the IFN- $\alpha$-inducible protein RIG-G was analyzed. RIG-G was consistently and strongly induced in both HUVEC and HDMEC by IFN- $\alpha$ (Figure 3). By contrast, no significant differences were found in the expression of cell cycle regulatory proteins. In HUVEC p21, p27, p53 and Cyclin D1 were slightly downregulated (Figure 3), whereas no effect at all was observed in HDMEC. Of the antiapoptotic proteins investigated only Mcl-1 was very slightly upregulated in HUVEC and HDMEC (Figure 3), whereas Bcl-2 and Bcl-x expression showed no consistent regulation in different experiments (not shown). Bak was slightly upregulated in most experiments in HDMEC but not HUVEC (Figure 3). Significant results were obtained with the inhibitor of differentiation Id-1, a protein associated with proliferation and negatively correlated to senescence and differentiation, ${ }^{30}$ and GBP-1, an inhibitor of proliferation. ${ }^{24}$ In both HUVEC and HDMEC Id-1 was consistently downregulated by IFN- $\alpha$, whereas GBP-1 was distinctly upregulated (Figure 3). These data demonstrate that IFN- $\alpha$ did not lead to a dramatic alteration of the expression of pro- or antiapoptotic proteins in EC. The fact that GBP-1 was upregulated and Id-1 was downregulated is consistent with the finding above that IFN- $\alpha$ did not induce proliferation of serum-starved cells. 

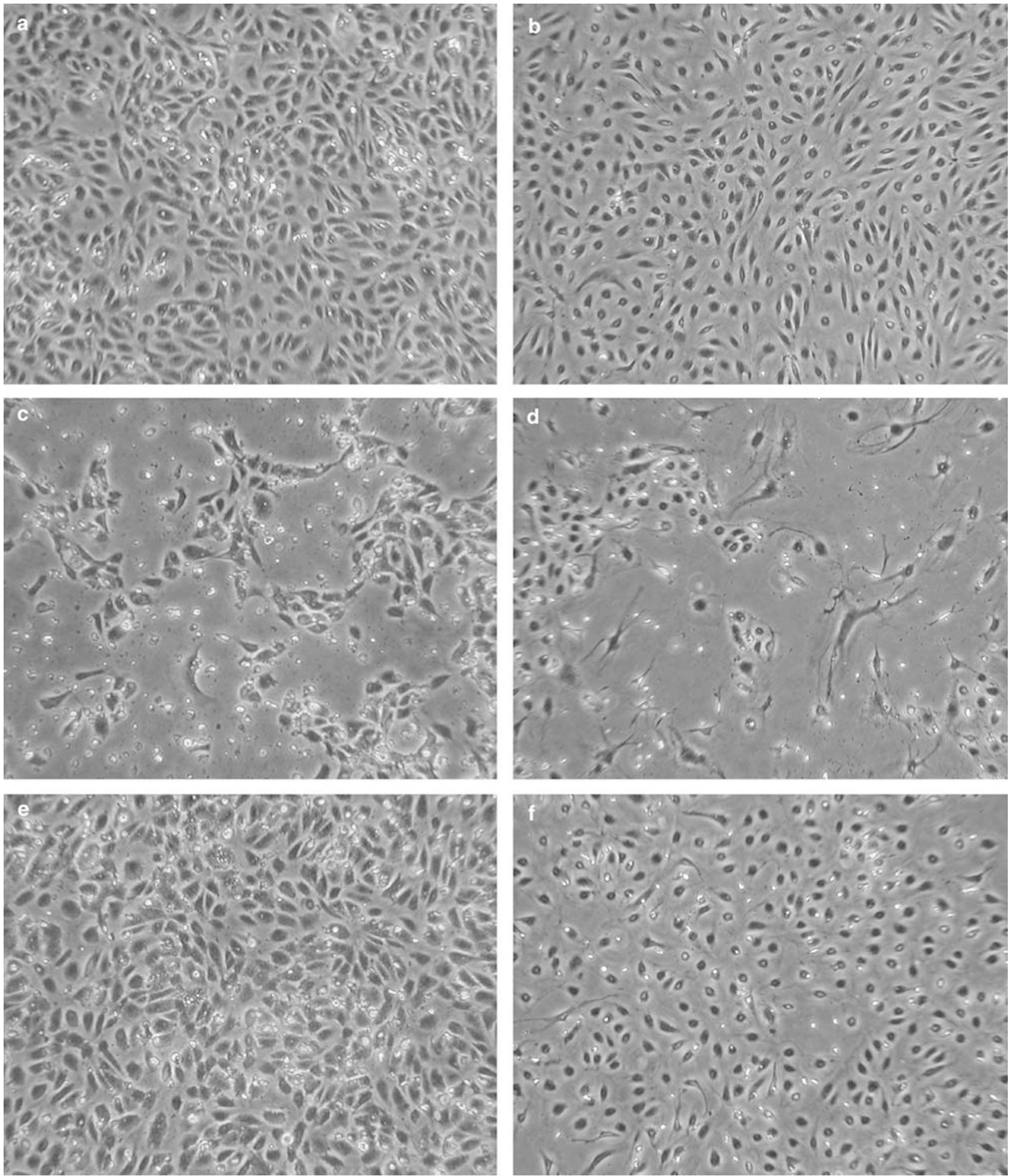

Figure 1 IFN- $\alpha$ protects endothelial cells from apoptosis induced by serum and growth factor withdrawal. HUVEC (a) and HDMEC (b) were grown to confluence in normal EC growth medium. Four days after switch of cultures to basal medium without growth factor and serum supplements, the vast majority of cells detached from the bottom of the culture vessels and showed morphological signs of apoptosis (c, d). Apoptosis was largely prevented when basal medium had been supplemented with IFN- $\alpha$ at $1000 \mathrm{U} / \mathrm{ml}(\mathbf{e}, \mathbf{f})$.

As IFN- $\alpha$ has been reported to activate the PI3K/ AKT pathway, ${ }^{31}$ which is implicated in prevention of apoptosis, ${ }^{32}$ we tested EC lysates for phophorylation of AKT. No increase of AKT phosphorylation in
HDMEC (Figure 4) was detected in six independent experiments 15,30 and $50 \mathrm{~min}$ after IFN- $\alpha$ stimulation, demonstrating that the PI3K/Akt pathway is not involved in the antiapoptotic effect of IFN- $\alpha$. 
Overexpression of GBP-1 Protects HUVEC from Serum-Deprivation-Induced Apoptosis

As GBP-1 was the only protein investigated that apart, from RIG-G, was reproducibly and distinctly regulated by IFN- $\alpha$ in both HUVEC and HDMEC, we tested its potential role for cell survival. Overexpression of GBP-1 in HUVEC resulted in a significant decrease in apoptosis after serum and growth factor deprivation (Figure 5). Protection against apoptosis was comparable to that of IFN- $\alpha$ treatment and could only be marginally augmented by the addition of IFN- $\alpha$ (Figure 5), indicating that the antiapoptotic effect of this cytokine is at least partly mediated by GBP-1. The effect of GBP-1 on
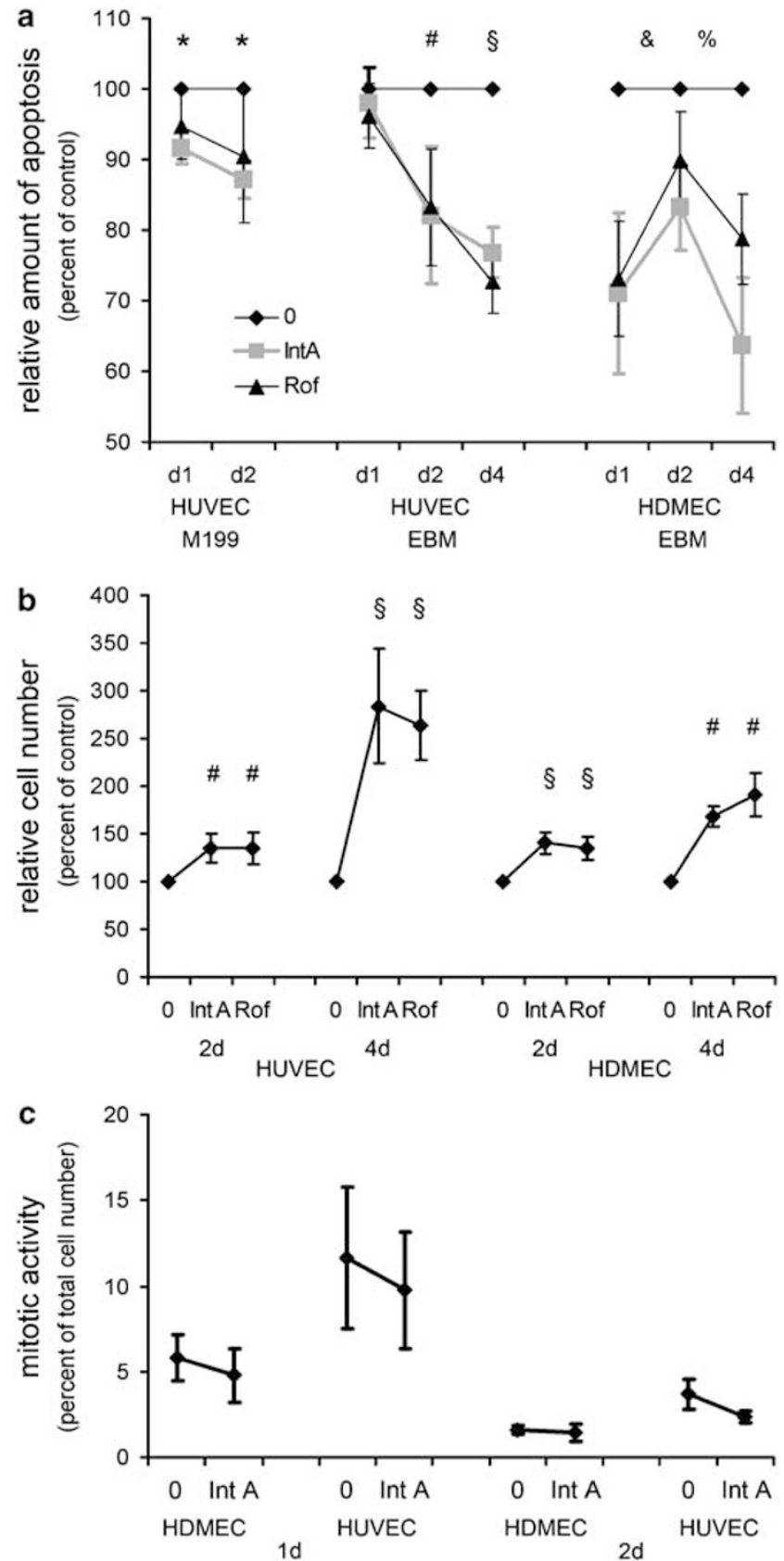

survival of HUVEC was also evident when the number of surviving cells was compared at different time points after seeding (Figure 6).

\section{Continuous Exposure to Interferon- $\alpha$ Reduces Population Doublings and Induces a Senescent Phenotype in HDMEC and HCAEC}

The antiapoptotic effect we observed with shortterm exposure of EC to IFN- $\alpha$ may be interpreted as

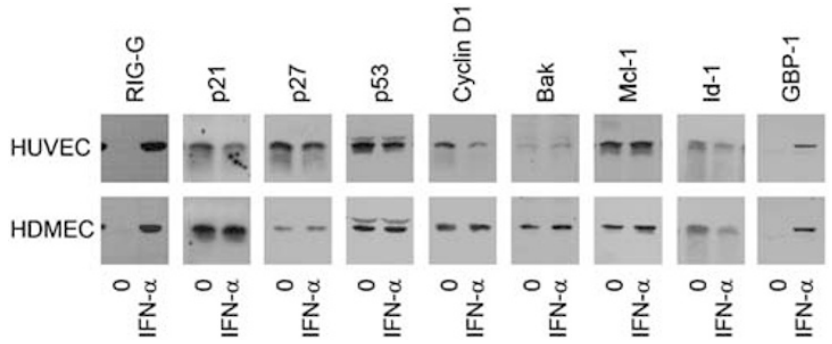

Figure 3 Effect of IFN- $\alpha$ on the expression of regulatory proteins of cell cycle and apoptosis. HUVEC (upper lane) and HDMEC (lower lane) were stimulated with IFN- $\alpha$ for $24 \mathrm{~h}$ in serum and growth factor-free medium. Western blot analyses were performed on whole cell lysates and repeated at least five times. RIG-G was strongly upregulated by IFN- $\alpha$ in all experiments and served as a positive control for IFN- $\alpha$ activity. In HUVEC a weak downregulation of p21, p27, p53 and Cyclin D1 by IFN- $\alpha$ was observed, which was not found in HDMEC. In both cell types a slight downregulation of Id-1 and a strong upregualtion of GBP-1 was regularly detected.

Figure 2 Effect of IFN- $\alpha$ on the rate of EC apoptosis, cell number and proliferation. (a) IFN- $\alpha$ reduces apoptosis of HUVEC and HDMEC. Apoptosis was quantified by measuring histone release into the supernatant on days 1,2 and 4 of EC culture under serum and growth factor-free conditions (culture medium M199 or EBM) with or without addition of IFN- $\alpha$ at $1000 \mathrm{U} / \mathrm{ml}$. The amount of histone release of cells grown without IFN- $\alpha$ was set as $100 \%$. Data are presented as mean value \pm s.e. IFN- $\alpha$ reduced the histone release of both HUVEC and HDMEC significantly. The effect was comparable for Intron A (IntA) and Roferon (Rof). *HUVEC $(n=6$, M199) d1: $P<0.002$ and d2: $P<0.0001$ for Int A (Tukey-HSD), d1 and d2: $P<0.043$ for Int A (Wilcoxon). ${ }^{~} \operatorname{HUVEC}(n=6,-$ ECGM) d2: $P<0.009$ for Int A, $P<0.007$ for Rof and d4: $P<0.001$ for Int A, $P<0.0001$ for Rof (Tukey-HSD). ${ }^{\S}$ HUVEC $(n=6,-$ ECGM) d4: $P<0.028$ for Int A and Rof (Wilcoxon). ${ }^{8}$ HDMEC $(n=6,-$ ECGM) d1: $P<0.0001$ for Int $A$ and Rof, d2: $P<0.023$ for Int A, d4: $P<0.0001$ for Int A and $P<0.002$ for Rof (Tukey-HSD). ${ }^{\%}$ HDMEC $(n=6,-$ ECGM) d1: $P<0.028$ for Int A and Rof, d2: $P<0.025$ for Int $\mathrm{A}, \mathrm{d} 4: \mathrm{P}<0.012$ for Int $\mathrm{A}$ and $P<0.025$ for Rof (Wilcoxon). (b) IFN- $\alpha$ treatment leads to increased EC survival after serum and growth factor withdrawal. EC survival was quantified by counting EC at different time points after serum and growth factor withdrawal. Stimulation of HUVEC and HDMEC with both Intron A and Roferon resulted in significantly higher cell numbers after 2 and 4 days when compared to untreated cells. The number of unstimulated cells was set as $100 \%$, data are presented as mean value \pm s.e. ${ }^{\S}(n=5): \quad P<0.046$ and ${ }^{\#}(n=6) \quad P<0.028$ for both HUVEC and HDMEC, Int $A$ and Rof (Wilcoxon). (c) IFN- $\alpha$ treatment does not induce EC proliferation. Mitotic activity of HDMEC and HUVEC grown in serum and growth factor-free medium was measured by assessing the proportion of Ki-67 positive cells $/ 100$ cells, (mean value \pm s.e.). Stimulation of both cell types with IFN- $\alpha$ resulted in a slight but not significant decrease of the mitotic activity when compared to untreated controls. HDMEC (1d: $n=6,2 \mathrm{~d}, n=5)$ and HUVEC (1d: $n=4,2 \mathrm{~d}$, $n=3)$ : NS. 
contradictory to its antiangiogenic activity in vivo. To mimic more closely an in vivo situation as it occurs in chronic inflammation where IFN- $\alpha$ is constantly present, we investigated the effects of continuous exposure to this cytokine.

When IFN- $\alpha$ was added every 2 days to fully supplemented culture medium of HDMEC and HCAEC, no distinct and reproducible morphologic differences were observed between treated and untreated cultures during the first few passages. However, after 2-4 weeks in medium supplemented with IFN $\alpha$, both HDMEC and HCAEC flattened,

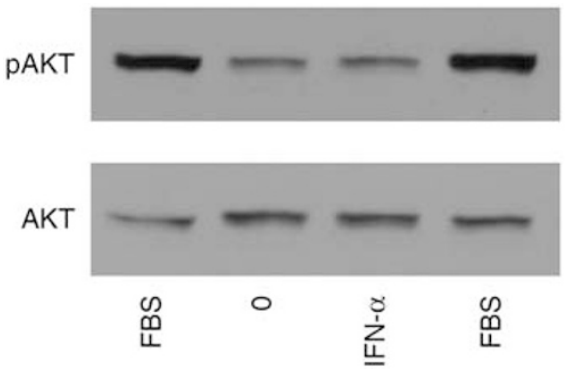

Figure $4 \mathrm{IFN}-\alpha$ treatment of HDMEC does not induce AKT phosphorylation. HDMEC grown in serum-free medium for $24 \mathrm{~h}$ were either exposed to $10 \%$ fetal bovine serum (FBS) or $1000 \mathrm{U} /$ ml IFN- $\alpha$ (Intron A). Western blot analysis with a monoclonal antibody recognizing phosphorylation of AKT at Ser473 $15 \mathrm{~min}$ after stimulation revealed that serum, but not IFN- $\alpha$, was able to induce AKT phosphorylation (upper panel). Total amount of AKT was equal in stimulated and control samples (lower panel). became irregular in shape with an increased diameter, thus exhibiting a phenotype characteristic for senescent EC (Figure 7a and b). The induction of cellular senescence by IFN- $\alpha$ was further confirmed

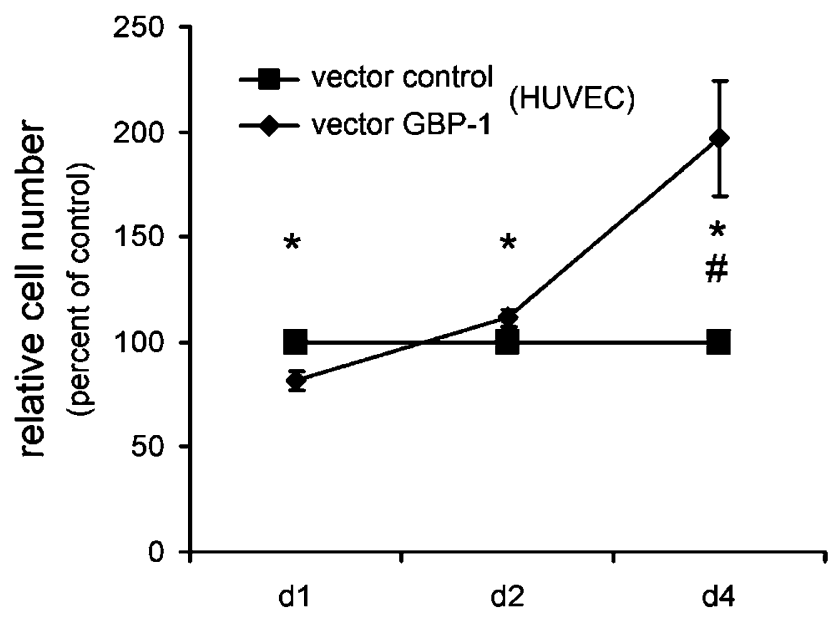

Figure 6 Overexpresion of GBP-1 enhances survival of HUVEC after serum and growth factor withdrawal. Survival of GBP-1 transduced (- -) and control (- $\mathbf{-}$-) HUVEC was quantified by counting EC 1, 2 and 4 days after serum and growth factor withdrawal. Numbers of control-transduced cells were set at $100 \%$ at every time point; data are presented as mean value \pm s.e. \#HUVEC $(n=8,-$ ECGM) d4: $P<0.001$ for control HUVEC vs GBP1 HUVEC (Tukey-HSD). *HUVEC $(n=8,-$ ECGM) d1: $P<0.017$; d2: $P<0.036$ and d4: $P<0.012$ for control HUVEC vs GBP-1 HUVEC (Wilcoxon).
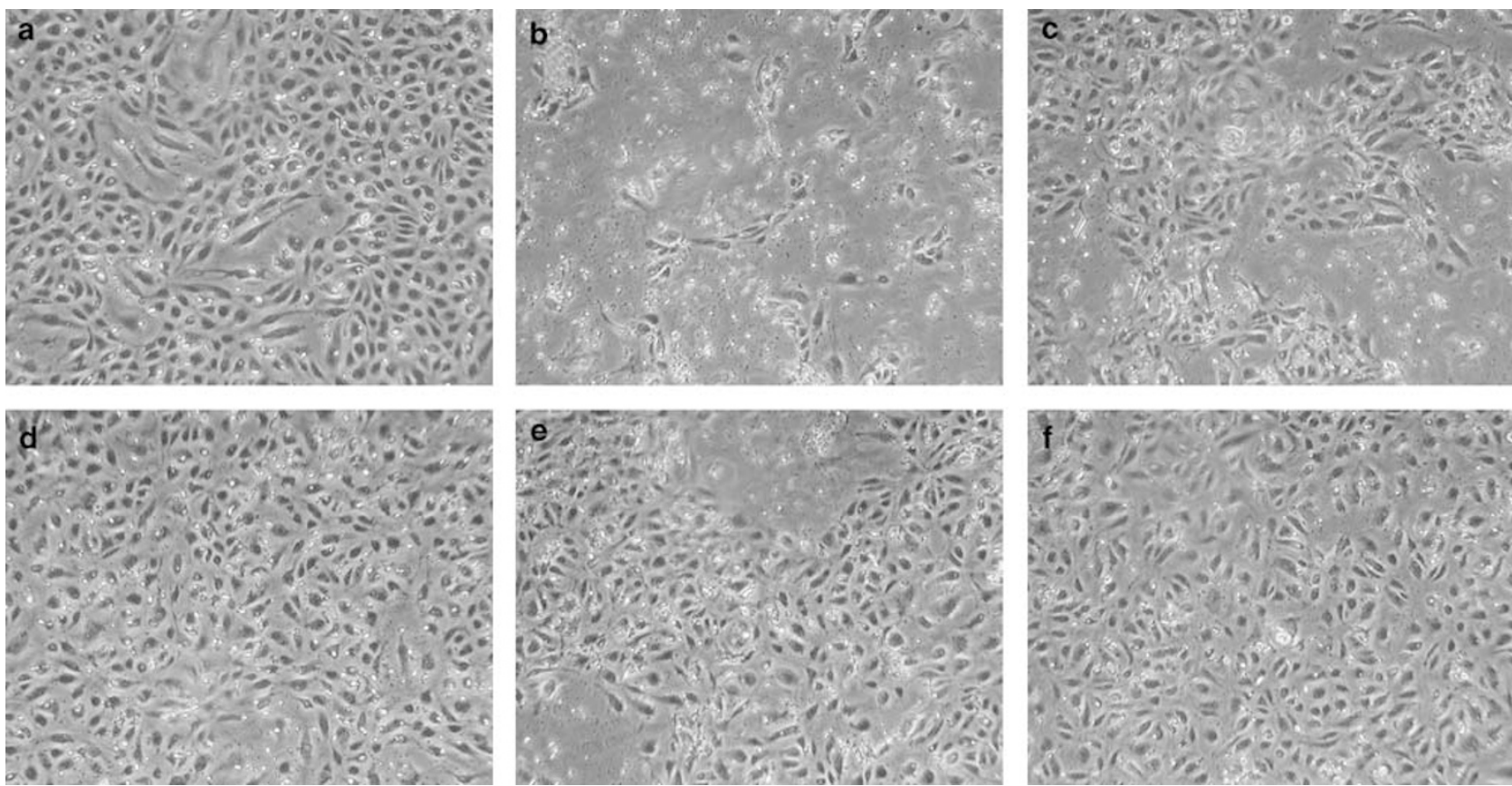

Figure 5 Transduction of HUVEC with GBP-1 protects HUVEC from apoptosis due to growth factor and serum withdrawal. HUVEC, stably transduced with either an empty expression vector (a-c) or with an vector expressing GBP-1 (d-f) were grown to confluency and subsequently cultured under serum and growth factor-free conditions. (a, d) Cells immediately after switching to serum and growth factor-free medium. Whereas control cells became apoptotic after 4 days (b), the vast majority of GBP-1 expressing cells (e) survived. Addition of IFN- $\alpha$ could prevent apoptosis of control cells (c) and also improved survival of GBP-1-transduced cells (f). 

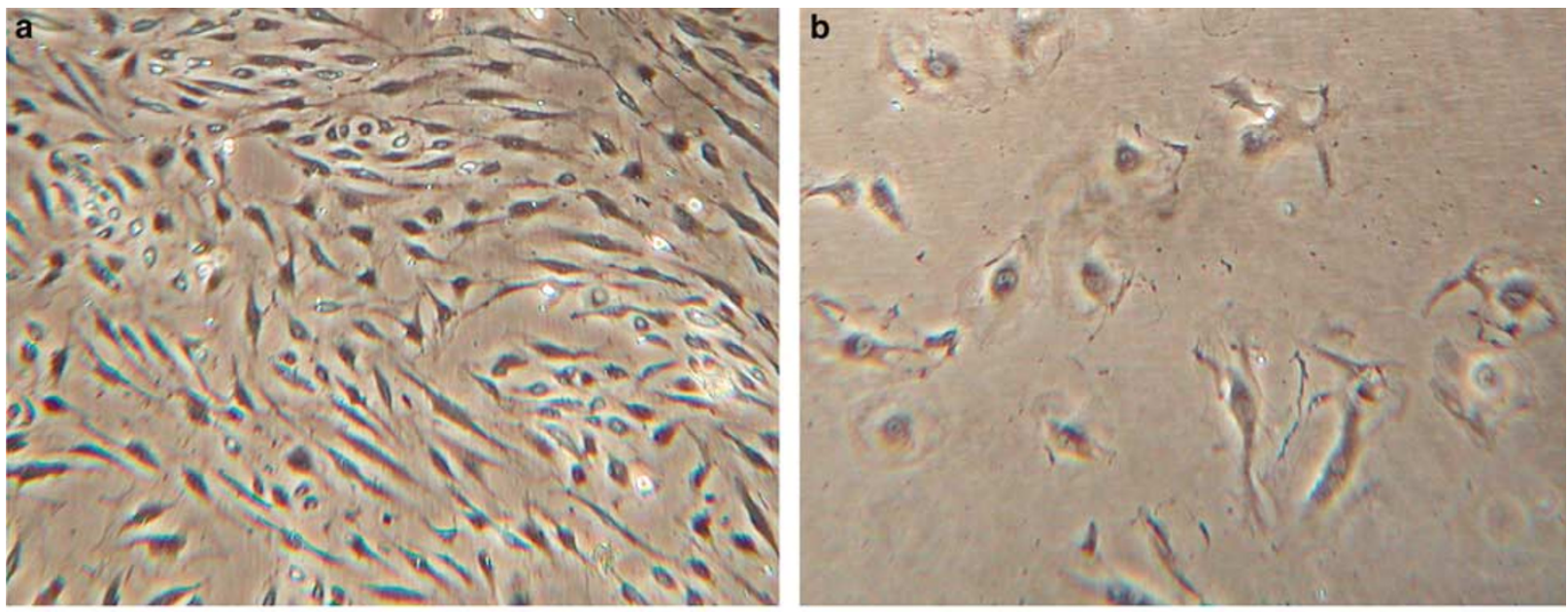

C

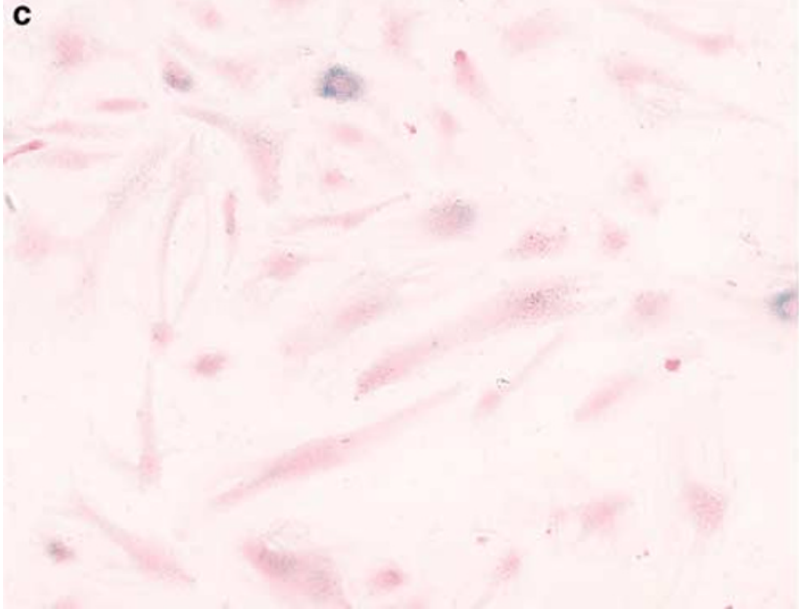

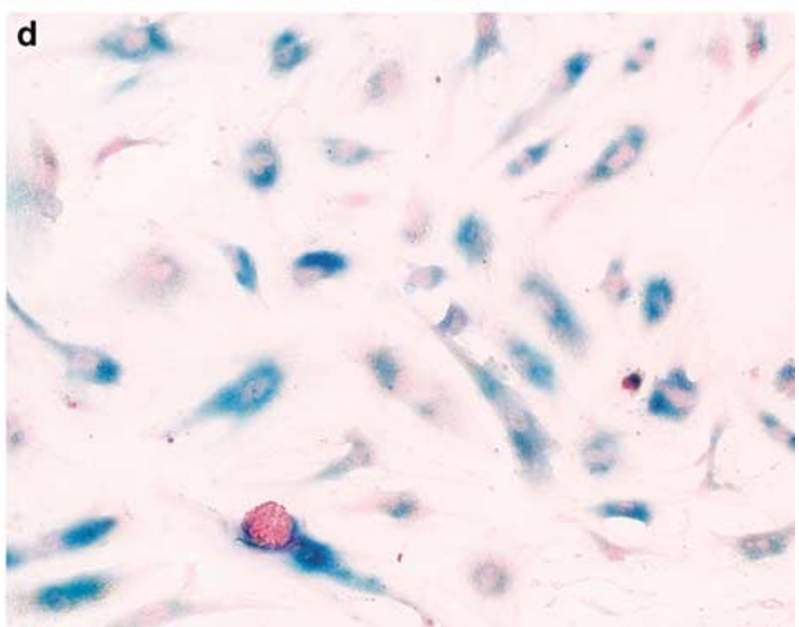

Figure 7 Continuous addition of IFN- $\alpha$ leads to premature senescence of EC. When IFN- $\alpha$ was added every second day to the EC cultures, HDMEC became enlarged, flattened and irregularly shaped corresponding to a senescent phenotype (b, passage 7) at passage numbers when cells grown without IFN- $\alpha$ were still inconspicuous (a, passage 7). As further indication of premature senescence of HDMEC after IFN- $\alpha$ treatement they showed a strongly increased expression of $\beta$-galactosidase (d, passage 14) as compared to control cells (c, passage 14).

by the finding that significantly more cells of the same passage expressed $\beta$-galactosidase in the treated (Figure $7 \mathrm{~d}$ ) as compared to the untreated cultures (Figure 7c). Podoplanin, a marker of lymphatic endothelial cells ${ }^{26}$ was detectable only on singly dispersed cells whereas the bulk of EC remained FVIII positive (not shown), confirming that no selection for non-blood vascular EC occurred during prolonged in vitro culture. On further culture, IFN- $\alpha$-treated cells stopped growing altogether, leading to a dramatically reduced number of population doublings in HDMEC and HCAEC (Figure 8). A reduction of population doublings was also observed in HUVEC, however, it did not reach statistical significance (Figure 8). In one of seven experiments using HCAEC, spindle-shaped EC derived from a culture of senescent IFN- $\alpha$-treated cells started to proliferate and eventually replaced the senescent cells. In this case, the final number of population doublings was higher in the stimulated cell cultures in contrast to all other experiments (not shown). When we analyzed the expression of Id-1 and GBP-1 proteins in long-term IFN- $\alpha$-treated EC, we found no regulation of the former, whereas a distinct upregulation of GBP-1 was consistently observed at all time points tested (Figure 9).

A typical functional feature of senescent EC is the enhanced binding of monocytes. ${ }^{33}$ When HDMEC or HCAEC were stimulated with IFN- $\alpha$ for 24 or $48 \mathrm{~h}$ no increased binding of freshly isolated human monocytes was observed when compared with untreated EC (not shown). When IFN- $\alpha$ treatment was continued for more than 10 population doublings, HCAEC showed a senescent phenotype and monocyte-adherence was distinctly increased in nine of 10 separate experiments (Figure 10). The same was observed in three of four experiments with senescent HDMEC (not shown). These data confirm that prolonged IFN- $\alpha$ treatment induces a premature senescent phenotype in blood vascular EC. As shortterm treatment of EC with IFN- $\alpha$ did not result in an increase of monocyte binding in accordance 
1004

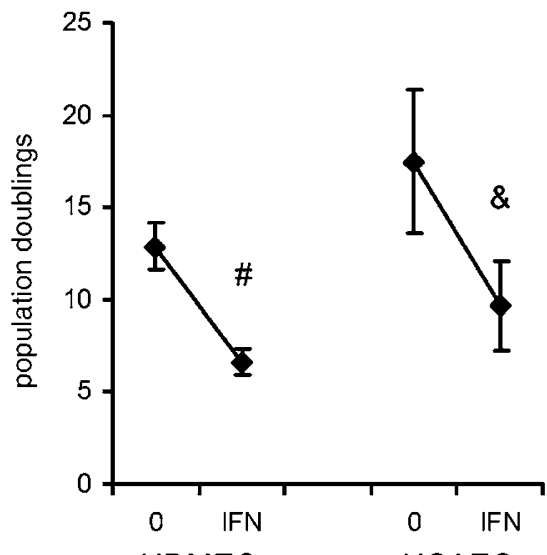

HDMEC

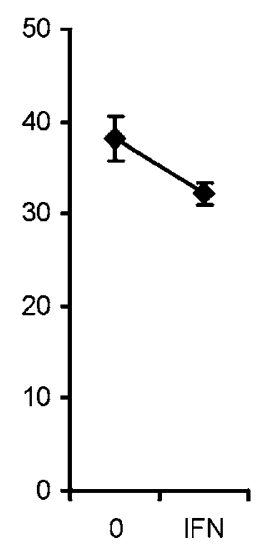

HUVEC

Figure 8 Continuous addition of IFN- $\alpha$ to EC cultures leads to a reduction of the number of population doublings in EC. HDMEC, HCAEC and HUVEC, grown in fully supplemented EC growth medium with or without addition of IFN- $\alpha$, were followed until they stopped to further divide. IFN- $\alpha$ resulted in a strong and significant decrease of the number of population doublings of both HDMEC and HCAEC (left panel) whereas the slight decrease of population doublings of HUVEC was not significant. "HDMEC $(n=12): P<0.002$; ${ }^{8}$ HCAEC $(n=6): P<0.028$; HUVEC $(n=4)$ : NS

a

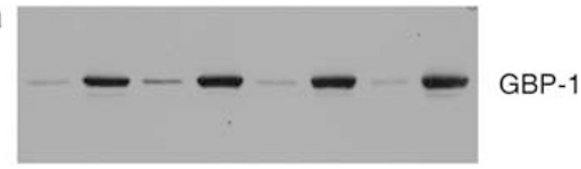

b

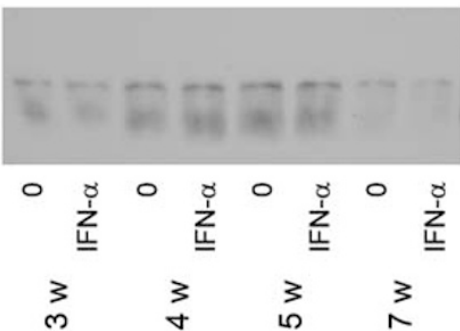

Figure 9 GBP-1 overexpression is maintained after long-term continuous addition of IFN- $\alpha$ to EC cultures. In analogy to shortterm exposure of HDMEC to IFN- $\alpha$ over $24 \mathrm{~h}$, long-term exposure of these cells grown in fully supplemented EC growth medium to IFN- $\alpha$ for 3 weeks to 7 weeks resulted in a strong upregulation of GBP-1 (a). In contrast to short-term IFN- $\alpha$ stimulated cells, no difference of Id-1 expression was observed between IFN- $\alpha$-treated and -nontreated HDMEC (b).

with previous studies, ${ }^{34,16}$ this binding effect is not caused by the direct cytokine-induced upregulation of adhesion molecules, but by their dysregulation due to senescence. ${ }^{33}$

\section{Discussion}

The antiangiogenic activities of IFN- $\alpha$ have been well documented in both animal models and clinical trials. Here, we studied the short-term and long-term effects of this cytokine on different types of EC in vitro. We found that short-term exposure to
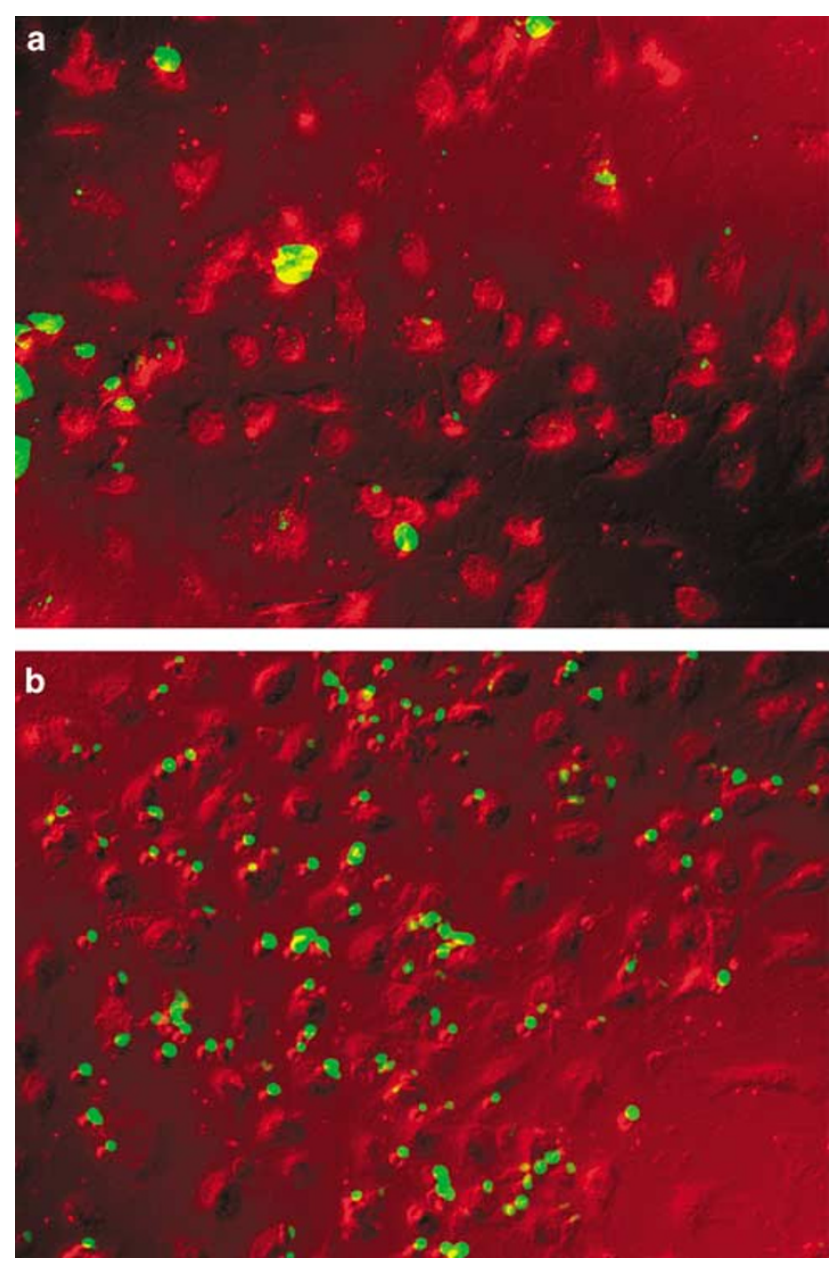

Figure 10 Chronic IFN- $\alpha$ treatment of HCAEC distinctly increased monocyte adhesion. Freshly separated monocytes were added to cultures of HCAEC grown either under standard conditions (a) or under continuous addition of IFN- $\alpha$ (b) for 10 passages. Whereas only few individual monocytes or monocyte clusters (a, green cells) bound to unstimulated HCAEC (a, red cells), high numbers of monocytes adhered to HCAEC continuously treated with IFN- $\alpha(b)$.

this cytokine prevents EC apoptosis after growth factor withdrawal and that long-term exposure induces replicative senescence in EC.

Our findings that IFN- $\alpha$ neither significantly inhibits nor induces growth of EC and does not only not induce apoptosis but rather acts antiapoptotically for EC contradicts previous studies which reported antiproliferative ${ }^{14-17}$ or promitotic, ${ }^{14,20,21}$ as well as proapoptotic ${ }^{22}$ activities. Unfortunately a direct comparison of our results with the ones of these studies is difficult if not impossible, since the experimental conditions were either vaguely described $^{14,22}$ or altogether different. ${ }^{20,21}$ Antiapoptotic activities of IFN- $\alpha$ in other cell types have been observed and related to activation of the PI-3 Kinase/AKT pathway. ${ }^{31}$ In our experimental setting, a participation of this pathway could be excluded since (1) no phosphorylation of AKT was observed after IFN-treatment, and (2) apoptosis of EC due to 
pharmacological inhibition of PI-3 kinase was reduced by IFN- $\alpha$ (data not shown). Also a modulation of antiapoptotic proteins Bcl-2, Bcl-x and Mcl-1 and proapoptotic proteins Bax and Bak by IFN- $\alpha$ appears not to play a role for the protective effect of IFN- $\alpha$ since we found no common effects on these regulators of apoptosis in HUVEC and HDMEC. By contrast, stable transfection of GBP-1, an IFNinducible protein ${ }^{24}$ which was consistently also upregulated under our experimental conditions, protected HUVEC against apoptosis comparably to IFN- $\alpha$ treatment. GBP-1 is a member of the family of large GTPases and has been shown to mediate the antiangiogenic effects of inflammatory cytokines on EC by inhibiting bFGF- and VEGF-induced proliferation and MMP-1 expression in EC. ${ }^{24,35}$ However, these activities cannot explain the GBP-1 antiapoptotic effect in serum-starved cells, where proliferation rates and MMP-1 expression are low. Of note, GBP-1 is a high turnover GTPase and continuous expression of this protein may induce a considerable shift of the cellular GTP pool. This may lead to a metabolically repressed EC phenotype, which may be less prone to stress-induced apoptosis by growth factor deprivation. Reduced cell reactivity of GBP-1 expressing cells to paracrine stimulation is in agreement with a less prominent increase of free intracellular calcium concentrations, which has been observed in constitutively GBP-1 expressing HUVEC after treatment with bFGF and VEGF (Sturzl, Pogoda, unpublished observations).

When IFN- $\alpha$ was added continuously to the cultures of HDMEC and HCAEC we observed a strong reduction of the number of their population doublings that is in agreement with the antiproliferative activity of GBP-1. By contrast, this effect was somehow lower in HUVEC again demonstrating differences in the biological responses of different EC types. ${ }^{36}$ Along with the reduction in population doubling, HDMEC and HCAEC acquired a phenotype characteristic for senescent EC, that is they became enlarged and started to express $\beta$-galactosidase more strongly and in a significantly higher proportion of cells than untreated populations. In contrast to EC exposed to IFN- $\alpha$ for a short time, these long-term treated cells also showed an increased adhesiveness for blood-derived monocytes.

For example, telomerase reverse transcriptase (hTERT) has been reported to be downregulated by IFN- $\alpha$ in T-cells and leukemic cell lines ${ }^{37}$ and IFI 16, a prosenescent scaffold protein necessary for the assembly of transcription factor compexes, ${ }^{38}$ is upregulated in fibroblasts ${ }^{39}$ and prostatic cell lines. ${ }^{38}$ Further studies will be necessary to exclude or confirm the involvement of these proteins in IFN$\alpha$ induced senescense of EC. As in short-term exposure, long-term exposure to IFN- $\alpha$ also resulted in strong expression of GBP-1. We have followed the GBP-1-transduced HUVEC and HUVEC transduced with a control vector for over four passages (cells stopped growing then) without observing a differ- ence in their morphology, suggesting that this enzyme might not be causally involved in this process (data not shown).

It might well be that the chronic administration of IFN- $\alpha$ for the treatment of hemangiomas or solid tumors induces senescence in EC in vivo analogous to our in vitro findings, and might thus contribute to its antiangiogenetic effects. ${ }^{10,11}$ Furthermore, premature endothelial senescence represents a critical step in the development of vasculopathies, ${ }^{40,41}$ for example, by decreasing proliferative capacity and increasing rates of apoptosis of aged endothelial cells and thereby contributing to functional impairment of the endothelial monolayer. ${ }^{42,43}$ If chronic production of IFN- $\alpha$ from endogenous sources plays a similar role in vivo as in vitro it might be one of the factors contributing to the induction or aggravation of atherosclerosis. It has been amply documented that atherosclerosis does not merely result from the accumulation of lipids due to hyperlipidemia but is associated with a variety of predisposing factors independent of lipid metabolism. ${ }^{44}$ Chronic inflammation, either locally or systemically, promotes the formation of atherosclerotic lesions. ${ }^{45-47}$ Inflammatory reactions which have been shown to increase the risk for atherosclerosis range from autoimmune diseases such as systemic vasculitides ${ }^{48}$ to microbial infections including cytomegalovirus (CMV), ${ }^{45}$ herpes simplex virus-1 (HSV-1), hepatitis A virus, ${ }^{49}$ Epstein-Barr virus (EBV), ${ }^{50}$ Chlamydia pneumoniae and Helicobacter pylori. ${ }^{46,47}$ These inflammatory reactions may occur at sites distant to those where atherosclerotic lesions form. ${ }^{46}$ IFN- $\alpha$ is strongly produced in several of these diseases ${ }^{4,51-53}$ and it is tempting to speculate that IFN- $\alpha$-induced EC senescence and resulting leucocyte adhesiveness could trigger or promote atherosclerotic lesions in the vessel walls. An additional argument for the involvement of IFN- $\alpha$ in pathogenesis of atherosclerotic is provided by recent work on LDL receptor-deficient mice in which chronic administration of IFN- $\alpha$ leads to the development of atherosclosis. ${ }^{54}$ The mode of action in this work has been explained by induction of hyperlipidemia. However, it is conceivable that a direct effect on EC might also have been operant in this system.

In conclusion, we have found that short-term exposure to IFN $-\alpha$ protects different types of EC against apoptosis and that the induction of GBP-1 is an important part of this protective effect. Chronic administration of this cytokine induces premature senescence and leukocyte adhesiveness of EC, a mechanism by which IFN- $\alpha$ might participate in the pathogenesis of atherosclerosis.

\section{Acknowledgements}

We wish to thank Heidemarie Rossiter for critically reading the manuscript. Research grants: German Cancer Aid (Deutsche Krebshilfe, Apoptose-Schwer- 
punktprogramm) and the interdisciplinary Center for Clinical Research (IZKF, projects A1 and B11) of the University of Erlangen to MS.

\section{References}

1 Gutterman JU. Cytokine therapeutics: lessons from interferon alpha. Proc Natl Acad Sci USA 1994;91: 1198-1205.

2 Gary-Gouy H, Lebon P, Dalloul AH. Type I interferon production by plasmacytoid dendritic cells and monocytes is triggered by viruses, but the level of production is controlled by distinct cytokines. J Interferon Cytokine Res 2002;22:653-659.

3 Stanley MA. Imiquimod and the imidazoquinolones: mechanism of action and therapeutic potential. Clin Exp Dermatol 2002;27:571-577.

4 Sedmak DD, Chaiwiriyakul S, Knight DA, et al. The role of interferon beta in human cytomegalovirusmediated inhibition of HLA DR induction on endothelial cells. Arch Virol 1995;140:111-126.

5 Rodel J, Assefa S, Prochnau D, et al. Interferon-beta induction by Chlamydia pneumoniae in human smooth muscle cells. FEMS Immunol Med Microbiol 2001;32:9-15.

6 Fujisawa H, Kondo S, Wang B, et al. The expression and modulation of IFN-alpha and IFN-beta in human keratinocytes. J Interferon Cytokine Res 1997;17: 721-725.

7 Di Bisceglie AM, Hoofnagle JH. Optimal therapy of hepatitis C. Hepatology 2002;36:S121-S127.

8 Santhanam S, Decatris M, O’Byrne K. Potential of Interferon-alpha in Solid Tumours: Part 2. BioDrugs 2002;16:349-372.

9 Lindner DJ, Kalvakolanu DV, Borden EC. Increasing effectiveness of interferon-alpha for malignancies. Semin Oncol 1997;24:S9-99-S99-104.

10 Garmendia G, Miranda N, Borroso S, et al. Regression of infancy hemangiomas with recombinant IFN-alpha 2b. J Interferon Cytokine Res 2001;21:31-38.

11 Wang L, Tang ZY, Qin LX, et al. High-dose and long-term therapy with interferon-alfa inhibits tumor growth and recurrence in nude mice bearing human hepatocellular carcinoma xenografts with high metastatic potential. Hepatology 2000;32:43-48.

12 De Bouard S, Guillamo JS, Christov C, et al. Antiangiogenic therapy against experimental glioblastoma using genetically engineered cells producing interferon-alpha, angiostatin, or endostatin. Hum Gene Ther 2003;14:883-895.

13 Stout AJ, Gresser I, Thompson WD. Inhibition of wound healing in mice by local interferon alpha/beta injection. Int J Exp Pathol 1993;74:79-85.

14 Pepper MS, Mandriota SJ, Vassalli JD, et al. Angiogenesis-regulating cytokines: activities and interactions. Curr Top Microbiol Immunol 1996;213:31-67.

15 Feldman D, Goldstein AL, Cox DC, et al. Cultured human endothelial cells treated with recombinant leukocyte A interferon. Tubuloreticular inclusion formation, antiproliferative effect, and 2', 5' oligoadenylate synthetase induction. Lab Invest 1988;58:584-589.

16 Ruszczak Z, Detmar M, Imcke E, et al. Effects of rIFN alpha, beta, and gamma on the morphology, proliferation, and cell surface antigen expression of human dermal microvascular endothelial cells in vitro. J Invest Dermatol 1990;95:693-699.

17 Minischetti M, Vacca A, Ribatti D, et al. TNP-470 and recombinant human interferon-alpha2a inhibit angiogenesis synergistically. Br J Haematol 2000;109: 829-837.

18 Mintzer RJ, Croze E, Rubanyi GM, et al. Differential effects of IFN-beta1b on the proliferation of human vascular smooth muscle and endothelial cells. J Interferon Cytokine Res 1998;18:939-945.

19 Bagavandoss P, Wilks JW. Isolation and characterization of microvascular endothelial cells from developing corpus luteum. Biol Reprod 1991;44:1132-1139.

20 Cozzolino F, Torcia M, Lucibello M, et al. Interferonalpha and interleukin 2 synergistically enhance basic fibroblast growth factor synthesis and induce release, promoting endothelial cell growth. J Clin Invest 1993;91:2504-2512.

21 Gomez D, Reich NC. Stimulation of primary human endothelial cell proliferation by IFN. J Immunol 2003;170:5373-5381.

22 Sgonc R, Fuerhapter C, Boeck G, et al. Induction of apoptosis in human dermal microvascular endothelial cells and infantile hemangiomas by interferon-alpha. Int Arch Allergy Immunol 1998;117:209-214.

23 Pammer J, Weninger W, Ban J, et al. The cell death regulatory protein bak is expressed in endothelial cells in inflamed tissues and Is induced by IFN-gamma in vitro. Biochem Biophys Res Commun 1999;264: 139-143.

24 Guenzi E, Topolt K, Cornali E, et al. The helical domain of GBP-1 mediates the inhibition of endothelial cell proliferation by inflammatory cytokines. EMBO J 2001;20:5568-5577.

25 Maciag T, Hoover GA, Stemerman MB, et al. Serial propagation of human endothelial cells in vitro. J Cell Biol 1981;91:420-426.

26 Breiteneder-Geleff S, Soleiman A, Kowalski H, et al. Angiosarcomas express mixed endothelial phenotypes of blood and lymphatic capillaries: podoplanin as a specific marker for lymphatic endothelium. Am J Pathol 1999;154:385-394.

27 Dimri GP, Lee X, Basile G, et al. A biomarker that identifies senescent human cells in culture and in aging skin in vivo. Proc Natl Acad Sci USA 1995;92: 9363-9367.

28 Lubeseder-Martellato C, Guenzi E, Jorg A, et al. Guanylate-binding protein-1 expression is selectively induced by inflammatory cytokines and is an activation marker of endothelial cells during inflammatory diseases. Am J Pathol 2002;161:1749-1759.

29 Gerber HP, McMurtrey A, Kowalski J, et al. Vascular endothelial growth factor regulates endothelial cell survival through the phosphatidylinositol 3'-kinase/ Akt signal transduction pathway. Requirement for Flk1/KDR activation. J Biol Chem 1998;273:30336-30343.

30 Norton JD. ID helix-loop-helix proteins in cell growth, differentiation and tumorigenesis. J Cell Sci 2000;113: 3897-3905.

31 Ruuth K, Carlsson L, Hallberg B, et al. Interferon-alpha promotes survival of human primary B-lymphocytes via phosphatidylinositol 3-kinase. Biochem Biophys Res Commun 2001;284:583-586.

32 Dimmeler S, Zeiher AM. Akt takes center stage in angiogenesis signaling. Circ Res 2000;86:4-5.

33 Matsushita H, Chang E, Glassford AJ, et al. eNOS activity is reduced in senescent human endothelial 
cells: Preservation by hTERT immortalization. Circ Res 2001;89:793-798.

34 Defazio G, Gelati $\mathrm{M}$, Corsini $\mathrm{E}$, et al. In vitro modulation of adhesion molecules, adhesion phenomena, and fluid phase endocytosis on human umbilical vein endothelial cells and brain-derived microvascular endothelium by IFN-beta 1a. J Interferon Cytokine Res 2001;21:267-272.

35 Guenzi E, Topolt K, Lubeseder-Martellato C, et al. The guanylate binding protein-1 GTPase controls the invasive and angiogenic capability of endothelial cells through inhibition of MMP-1 expression. EMBO J 2003;22:3772-3782.

36 Cines DB, Pollak ES, Buck CA, et al. Endothelial cells in physiology and in the pathophysiology of vascular disorders. Blood 1998;91:3527-3561.

$37 \mathrm{Xu} \mathrm{D}$, Erickson S, Szeps $\mathrm{M}$, et al. Interferon alpha down-regulates telomerase reverse transcriptase and telomerase activity in human malignant and nonmalignant hematopoietic cells. Blood 2000;96:4313-4318.

38 Xin H, Curry J, Johnstone RW, et al. Role of IFI 16, a member of the interferon-inducible p200-protein family, in prostate epithelial cellular senescence. Oncogene 2003;22:4831-4840.

39 Xin H, Pereira-Smith OM, Choubey D. Role of IFI 16 in cellular senescence of human fibroblasts. Oncogene 2004;23:6209-6217.

40 Minamino T, Miyauchi H, Yoshida T, et al. The role of vascular cell senescence in atherosclerosis: antisenescence as a novel therapeutic strategy for vascular aging. Curr Vasc Pharmacol 2004;2:141-148.

41 Brodsky SV, Gealekman O, Chen J, et al. Prevention and reversal of premature endothelial cell senescence and vasculopathy in obesity-induced diabetes by ebselen. Circ Res 2004;94:377-384.

42 Hoffmann J, Haendeler J, Aicher A, et al. Aging enhances the sensitivity of endothelial cells toward apoptotic stimuli: important role of nitric oxide. Circ Res 2001;89:709-715.

43 Wagner M, Hampel B, Bernhard D, et al. Replicative senescence of human endothelial cells in vitro involves G1 arrest, polyploidization and senescence- associated apoptosis. Exp Gerontol 2001;36:13271347.

44 Epstein SE, Zhou YF, Zhu J. Infection and atherosclerosis: emerging mechanistic paradigms. Circulation 1999;100:e20-e28.

45 Muhlestein JB, Anderson JL. Chronic infection and coronary artery disease. Cardiol Clin 2003;21:333-362.

46 Rupprecht HJ, Blankenberg S, Bickel C, et al. Impact of viral and bacterial infectious burden on long-term prognosis in patients with coronary artery disease. Circulation 2001;104:25-31.

47 Georges JL, Rupprecht HJ, Blankenberg S, et al. Impact of pathogen burden in patients with coronary artery disease in relation to systemic inflammation and variation in genes encoding cytokines. Am J Cardiol 2003;92:515-521.

48 Numano F. Vasa vasoritis, vasculitis and atherosclerosis. Int J Cardiol 2000;75:S1-S8; discussion S17-S19.

49 Zhu J, Nieto FJ, Horne BD, et al. Prospective study of pathogen burden and risk of myocardial infarction or death. Circulation 2001;103:45-51.

50 Shi Y, Tokunaga O. Herpesvirus (HSV-1, EBV and $\mathrm{CMV}$ ) infections in atherosclerotic compared with non-atherosclerotic aortic tissue. Pathol Int 2002;52: 31-39.

51 Eloranta ML, Sandberg K, Alm GV. The interferonalpha/beta responses of mice to herpes simplex virus studied at the blood and tissue level in vitro and in vivo. Scand J Immunol 1996;43:356-360.

52 Kaukoranta-Tolvanen SS, Teppo AM, Laitinen K, et al. Growth of Chlamydia pneumoniae in cultured human peripheral blood mononuclear cells and induction of a cytokine response. Microb Pathog 1996;21: 215-221.

53 Hausfater P, Fillet AM, Rozenberg F, et al. Prevalence of viral infection markers by polymerase chain reaction amplification and interferon-alpha measurements among patients undergoing lumbar puncture in an emergency department. J Med Virol 2004;73:137-146.

54 Fabricant CG, Fabricant J. Atherosclerosis induced by infection with Marek's disease herpesvirus in chickens. Am Heart J 1999;138:S465-S468. 